\title{
Classification of manipulators of the same origin by virtue of compactness and complexity
}

\author{
Erkin Gezgin*, Serhan Ozdemir \\ Artificial Iytelligence E' Design Lab, Mechanical Engineering Department, Izmir Institute of Technology, 35437 Izmir, Turkey
}

\section{A R T I C L E I N F O}

\section{Article history:}

Received 10 May 2011

Accepted 18 May 2011

Available online 24 June 2011

\section{Keywords:}

Compactness

Complexity

Entropy

Robot manipulators

Classification

\begin{abstract}
A B S T R A C T
This work deals with a classification method that employs concepts such as complexity and compactness. The idea is to classify manipulators, or any other mechanism for that matter, of the same origin, based on the geometry of the joints, the tasks performed by the joints, the efficiency and the manufacturing cost to generate the specified efficiency. It is known that successive units on a single branch create individual uncertainties that affect the eventual quality of the performed operation [1]. An entropic expression quantifies this uncertainty in terms of the number of links and the unit effectiveness. The concepts of compactness and complexity have been formulated, and these concepts are explained through serial and parallel manipulators with varying parameters. Eventually, a cost function is created which is a function of complexity, uncertainty and the manufacturing cost. A worked example on $\mathrm{M}=6$ StewartGough platform is given how this cost function could be taken advantage of when deciding an initial manipulator. A genetic algorithm is used for the optimization of the cost function, where the results are tabulated.
\end{abstract}

(c) 2011 Elsevier Ltd. All rights reserved.

\section{Introduction}

An important step in the design of robot manipulators is the structural synthesis concept. The structural synthesis provides the calculation of the desired degrees of freedom of the robot manipulators. Starting with Euler in the mid 18th century, many formulations have been created to satisfy the calculations of different types of manipulators. By the introduction of new parameters and methods of the structural synthesis, novel methods were generated to categorize manipulators.

Of the most relevant literature worth mentioning, one could start with Malushev. Malushev [2] introduced a universal mobility equation with respect to the number of links and kinematic pair classes. The history of kinematic structures and structural synthesis is reviewed by Mruthyunjaya [3], and the history of structural formulations is presented as a table with equations, authors, years and some commentaries along with the description of the structural synthesis of serial platform manipulators by Alizade et al. [4]. Mobility equations without exception for mechanisms, which contain mixed independent loops with variable general constraints, are given by Freudenstein et al. [5]. A new structural formulation is proposed by Alizade [6] for the mechanisms with the same independent loops in the same space or subspace, where mobile platform types, number of mobile platforms, and number of connections between the platforms were included along with mobility of kinematic pairs. Introducing a new formulation of the number of independent loops, the structural synthesis of parallel manipulators is investigated by Alizade et al. [7]. In this study, structural groups of the serial platform robot manipulators are shown in tables for subspace $\lambda=3$ and space $\lambda=6$.

In the light of the proposed theories, an increase in new robot manipulators and kinematic structures is observed recently. So that, in the field of mechanisms, classification ideas become important for the ease of selection and identification. Balkan et al. [8]

\footnotetext{
* Corresponding author. Tel.: +90 232750 6752; fax: +90 2327506701 .

E-mail address: erkingezgin@iyte.edu.tr (E. Gezgin).
} 
introduced a kinematic structure based classification of six degrees of freedom industrial robot manipulators. Lin et al. [9] presents a classification method for input joints of planar five bar two degrees of freedom mechanisms or in-parallel manipulators. The classification method classifies the input joints of all five bar mechanisms into four classes. Su and McCarthy et al. [10] classifies the movement of the RRSS spatial linkage in terms of its link dimensions. Cervantes-Sanchez et al. [11] introduced a robust classification scheme for the 4R spherical linkages.

As for the idea of uncertainty, entropy is the perfect tool. Entropy has been in use for decades in a wide range of areas. It has its origins back in the 19th century, dating back to Boltzmann. His formulation of entropy was meant to quantify the disorder that every physical system strives to maximize. Considered as the cornerstone in statistical mechanics, it has been put to use from black holes to chemical mixtures.

In the context of mechanisms, the uncertainty emerges when the links and the joints are considered as imperfect constituents. Each joint in series is thought to be a source of uncertainty. This uncertainty would reflect the designer's trust in the transmission as joint efficiency, which may be expressed in an entropy related expression. To clear the issue further, one must understand that a constant of efficiency is nothing but an average value of previous experiments or a catalogue value of the manufacturer. This is to say that under all conditions, a certain percentage of power will always be transferred. But this is an oversimplification. Alongside this constant, a term of uncertainty also ought to be given. Considering the fact that power transmission is interplay of many factors, from temperature to lubrication etc., transmission phenomenon is converted into a stochastic event that is expressed in entropy. Logarithmic formulation of uncertainty penalizes low efficiencies further than simple algebraic operations would do. This is especially important when motion is split, and each branch has multiple units. Such a design, and a qualitative elaboration could be found in Ozdemir [1,12] respectively.

Compactness and complexity are the two terms that are often used in manipulator or mechanism descriptions. These terms are subjective and intangible qualifications, still yet one finds such qualifications in mechanism research. When a mechanism or a machine is called compact (or complex), there are no solid guidelines to test against other mechanisms in terms of compactness. For example, Son et al. [13] define parallel milling machines more compact compared to serial ones. Kim and Cho [14] also claim the compactness of their micro stage for vibration control. Another similar claim is found in [15], where Gonzales et al. mention the compactness of the mechanism assisting climbing of obstacles, without any firm reference to the description of compactness or complexity as a formulated entity. There are some indices used in mechanism synthesis which may or may not sound abstract such as sensitivity as in [16] formulated for planar parallel mechanisms. It is rather unfortunate that the people working in this area do not have a compactness measuring stick to soundly quantify the compactness and the complexity of their designs against their peers. We believe that this manuscript has firmly put the feet of the compactness on the ground.

\section{Compactness and complexity formulas}

A wide range of robotic mechanical systems consists of parallel platform manipulators, serial platform manipulators, multiple serial chains, and hybrid robotic mechanical systems. All platform robotic mechanical systems constructed from the actuators and simple structural groups are made up of one or more platforms, legs, branches and hinges. If two separate robot manipulators are said to be from the same origin, then it can be said that they are generated by using the same structural group for the desired mobility where the total number of pairs and links change while the other parameters such as the number of platforms, actuators, legs and hinges remain constant. As a result, the identification of these manipulators can only be made by using their pair or link numbers. Due to the fact that the total degrees of freedom of the pairs do not change in the same originated manipulators, link numbers are easy to compute for the distinction purposes when compared with the pairs.

The number of links of a robot manipulator can easily be computed by using mobility formulation that can be extracted from Somov-Malushev's universal mobility formula,

$$
M=\lambda(l-1)-\sum_{i=1}^{4}(\lambda-i) P_{i}
$$

where $M$ is the mobility, $\lambda$ is the general space or subspace, $l$ is the number of links of the manipulator, and $P_{i}$ represents the number of $i$ th DoF pairs. Using Eq. (1) the number of links $l$ can be calculated as,

$$
l=\frac{1}{\lambda}\left(M+\sum_{i=1}^{4}(\lambda-i) P_{i}\right)+1
$$

As the number of links $l$ alone will not give any comparative information, we can define a new distinguishing parameter Compactness $(L)$. The compactness reveals the information about the current configuration and the selected robot manipulator with its most primitive origin that is the one having the maximum possible number of links. By definition, the compactness can be formulated as,

$$
L=l_{\max }-l
$$


Table 1

Classification of $M=1$ seven bar mechanism in $\lambda=6$.

\begin{tabular}{|c|c|c|c|c|c|c|c|c|c|}
\hline & Manipulator & $P_{i}$ & $L$ & $C$ & & Manipulator & $P_{i}$ & $L$ & $C$ \\
\hline 1 & 8 & $7 P_{1}$ & 0 & 1 & 3 & h & $4 P_{1} 1 P_{3}$ & 2 & 0.33 \\
\hline 2 & & $5 P_{1} 1 P_{2}$ & 1 & 0.5 & 4 & & $2 P_{1} 1 P_{2} 1 P_{3}$ & 3 & 0.25 \\
\hline
\end{tabular}

Using Eq. (2), and the case that the most primitive of any manipulator originating from the same structural group is just composed of one degree of freedom pairs, $P_{1}=\sum f_{i}$, the maximum link number will be,

$$
l_{\max }=\frac{1}{\lambda}\left(M+(\lambda-1) P_{1}\right)+1
$$

and by combining the Eqs. (2), (3) and (4), the general compactness formulation can be given as,

$$
L=\frac{1}{\lambda}\left((\lambda-1) \sum f_{i}-\sum_{i=1}^{4}(\lambda-i) P_{i}\right)
$$

Eqs. (3), and (5) state that the compactness of the manipulators increase when their number of links decrease by using the exchangeability of kinematic pairs. Also note that the zero compactness refers to the most primitive manipulator of the selected origin.

After describing the compactness concept, another similar parameter can be introduced as Complexity $(C)$. The complexity can be described as an index, which could be scaled between zero and one. Complexity approaches zero as the manipulators become compacter. If the complexity of the least compact manipulator of the selected origin is unity, then the most compact form will tend to zero. Using this convention, the complexity can be formulated as,

$$
C=\frac{1}{L+1}
$$

where $L$ is the compactness of the selected manipulator. Eq. (6) can be expanded with Eq. (5) as,

$$
C=\left[\frac{1}{\lambda}\left[(\lambda-1) \sum f_{i}-\sum_{i=1}^{4}(\lambda-i) P_{i}\right]+1\right]^{-1}
$$

Tables 1 through 4 show the compactness and complexity classification examples of different robot manipulators in space, $\lambda=6$, and Fig. 1 shows the complexity and compactness relationship.

Table 2

Classification of $M=6$ serial robot manipulator in $\lambda=6$.

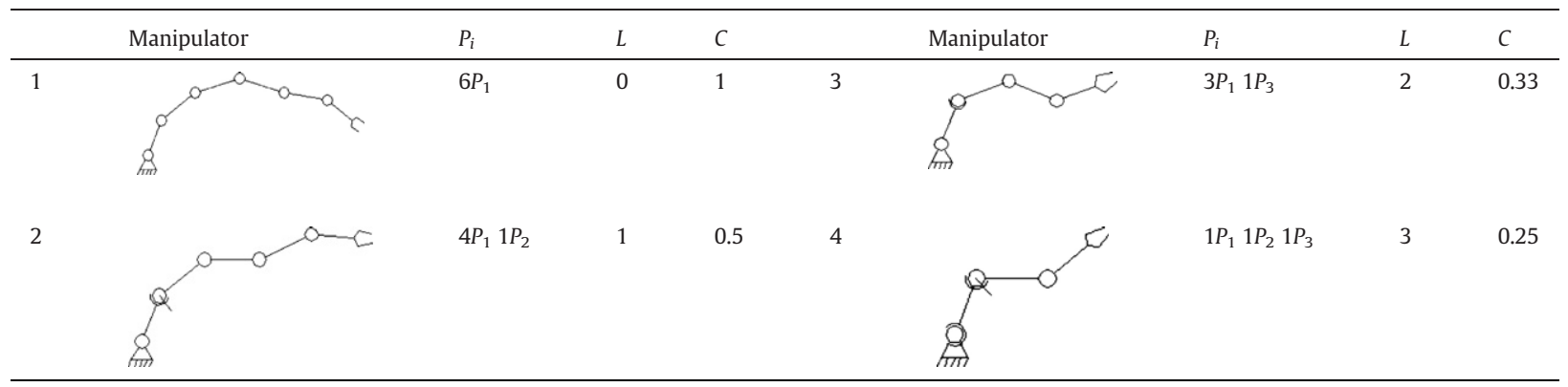


Table 3

Classification of 6 mobility triangular parallel platform robot manipulator in $\lambda=6$.

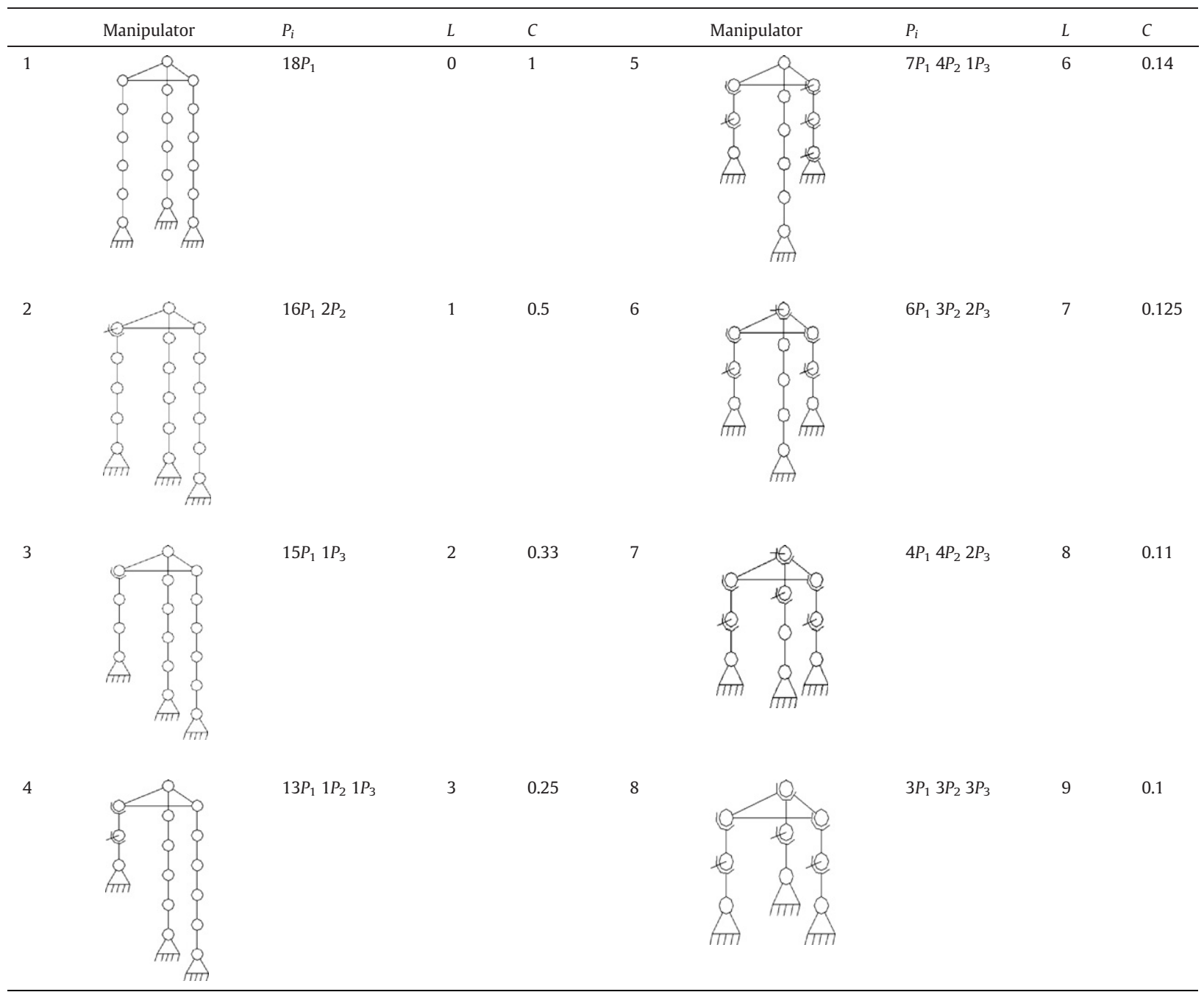

\section{Classification of robot manipulators with respect to the pair efficiencies through entropy}

Having set up the convention and the definition of compactness and complexity, the concept can be extended by uncertainties. From this point of view, entropy is a convenient tool that may quantify the imperfections of joints. Entropy can be formulated as [1],

$$
S=-\sum_{i=1}^{m} P_{i} \log P_{i}
$$

where, $P_{i}$ is the probability that the motion could be transmitted through the element $i$.

If the case is adapted to the robot manipulators, kinematic pair efficiencies of robot manipulators are selected as the motion transmission probabilities. Since the specified efficiencies are not constants, as stated before, transmission of motion is turned to a stochastic phenomenon. Owing to this, Eq. (8) can be rewritten as,

$$
S=-\sum_{i=1}^{j} \eta_{i} \log \eta_{i}
$$

where, $\eta_{i}\left(0<\eta_{i}<1\right)$ is the efficiency of the $i$ th kinematic pair of the robot manipulator and $j$ is the number of kinematic pairs. If all the pair efficiencies are assumed to be the same $(\eta)$ then Eq. (9) will become,

$$
S=-j(\eta \log \eta)
$$


Table 4

Classification of $\mathrm{M}=4$ double triangular serial platform robot manipulator in $\lambda=6$.

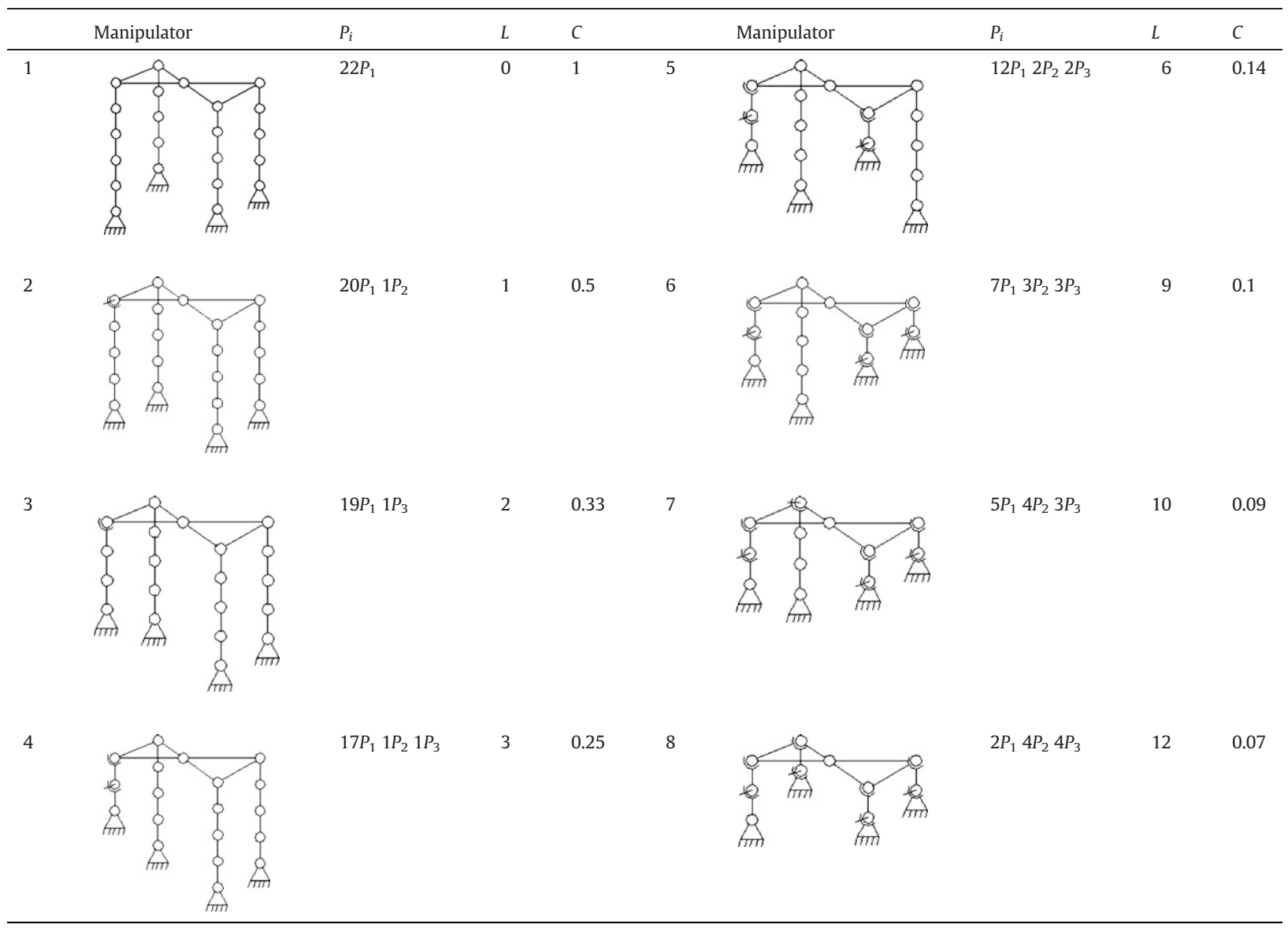

However, to use the entropy clearly for classification, Eq. (10) should be normalized to constrain the classification parameter between zero and one. Now call $f(\eta)=-\eta \log \eta$, so that the maximum possible value of Eq. (10) will bef( $\eta)_{\max } \cdot j_{\max }$, and the normalized entropy $\left(S_{n}\right)$ can be written as,

$$
S_{n}=\frac{-j\left(\eta_{i} \log \eta_{i}\right)}{f(\eta)_{\max } \cdot j_{\max }}
$$

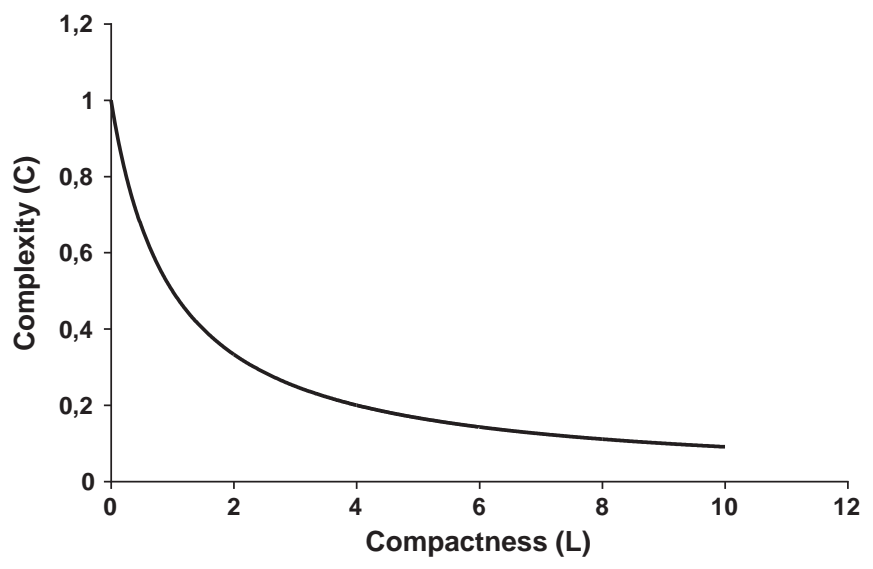

Fig. 1. Complexity and compactness relationship. 
Note that, when the indeterminacies are at a maximum, nothing could be said about the situation of the robot manipulator, whether it will make the desired action or not, since the maximum uncertainty reigns. As the efficiencies increase, the uncertainty regarding the operation will decrease. To keep the entropy low with higher efficiencies, a threshold is selected for the simulations at an efficiency of 0.4 , which constitutes the lower limit.

Owing to the fact that the normalized entropy, Eq. (11), is always between zero and one, it can be used as a universal indicator for the quality of the job done. As stated before, if the normalized entropy approaches zero, no uncertainty is created regarding the motion transmission, where apparently all the joint efficiencies are 1 . However, as the normalized entropy approaches one, it points to the fact that maximum uncertainty is generated for the given configuration.

Now, as an example of classification and design, first, let us assume that the desired normalized entropy of a robot manipulator for some predetermined task will be $S_{n}=0.2$, and 6 mobility triangular parallel platform robot manipulator in $\lambda=6$ is to be used. Table 3 can be re-tabulated with respect to the types of the manipulators and their minimum reachable pair efficiencies for the desired entropy (Table 5, Column A). It is easily seen that, as the robot manipulators get compacter, minimum usable pair efficiencies decreased. Most clearly, compacter manipulators allow us to select the kinematic pairs from a large variety of efficiencies for the selected entropy, while in the complex manipulators, selections are bound to the higher efficiencies.

Table 5

$\mathrm{M}=6$ triangular parallel platform robot manipulator in $\lambda=6$. Column A) Minimum reachable pair efficiencies with $S_{n}=0.2$. Column B) Entropies with pair efficiencies $\eta=0.9$.

\begin{tabular}{|c|c|c|c|c|c|c|c|c|c|}
\hline & & & A & B & & & & A & B \\
\hline & Manipulator & $j$ & $\eta_{i \min }$ & $S_{n}$ & & Manipulator & $j$ & $\eta_{i \min }$ & $S_{n}$ \\
\hline 1 & & $j_{\max } 18$ & 0.923 & 0.257 & 5 & नाता & 12 & 0.882 & 0.171 \\
\hline 2 & & 17 & 0.918 & 0.243 & 6 & & 11 & 0.870 & 0.157 \\
\hline 3 & & 16 & 0.913 & 0.229 & 7 & & 10 & 0.856 & 0.143 \\
\hline 4 & & 15 & 0.907 & 0.214 & 8 & & 9 & 0.839 & 0.128 \\
\hline
\end{tabular}


As a second example, this time on the 6 mobility triangular parallel platform robot manipulator in $\lambda=6$, pair efficiencies will be fixed to the value $\eta=0.9$ and again Table 3 is re-tabulated with respect to the normalized entropies (Table 5, Column B). Note that in this situation, the effect of the compactness is much clearer. From the most complex robot manipulator to the most compact one, nearly a hundred percent decrease in normalized entropy is observed.

Please note that less number of joints allow the use of less efficient joints to give the same overall uncertainty. This is reflected in the budget considerations. Or conversely, for the same level of efficiency for all joints, compacter versions generate less entropy.

\section{Compactness and pair efficiency optimization for $M=6$ Stewart-Gough platform by using genetic algorithms}

A genetic algorithm (GA) is a search technique often used in computational intelligence. GAs, roughly speaking, are both guided and random, and are categorized as global search heuristics. GAs are a particular class of evolutionary algorithms that use techniques inspired by evolutionary biology such as inheritance, mutation, selection, and crossover. They are used as a computer program in which a population of solutions to an optimization problem evolves toward better solutions. More could be found in Munakata [17].

After the specifications of the terms, compactness, complexity and normalized entropy for the robot manipulators, genetic algorithms are used for the optimization. In this case, the aim is to find the best mechanism from the selected class that fulfills the desired task with respect to the restrictions. The main part of the algorithm is the selection of the cost function, which should be minimized in order to meet the constraints. The cost function is carefully selected so that it contains compactness, entropy and the manufacturing cost parameters at the same time with certain modifying factors. Note that, compactness still includes complexity itself, so that it is not necessary to use additional complexity constraints in the function. The selected cost function can be formulated as,

$$
J=L+S+c
$$

where $c$ represents the manufacturing cost of the mechanism, and formulated as,

$$
c=K P_{C} j
$$

where $P_{C}$ is the raw cost. It is the minimum investment cost of one pair for any producer in order to start production of the kinematic pairs and $K$ is the modification function that can be introduced as the multiplication of the penalty functions of pair efficiency and compactness respectively as,

$$
K=k_{\eta} k_{L}
$$

As the pair efficiencies approach one, due to the production precision and quality, the total cost should increase by the amount of penalty efficiency function. After many trials and observations of the results, this penalty function is shaped as,

$$
k_{\eta}=\sqrt{1 / \log (1 / \eta)}
$$

It can be seen in Fig. 2 that Eq. (15) will tend to go infinity for the efficiencies that are too close to one, which means for higher efficiencies, higher penalties will be paid.

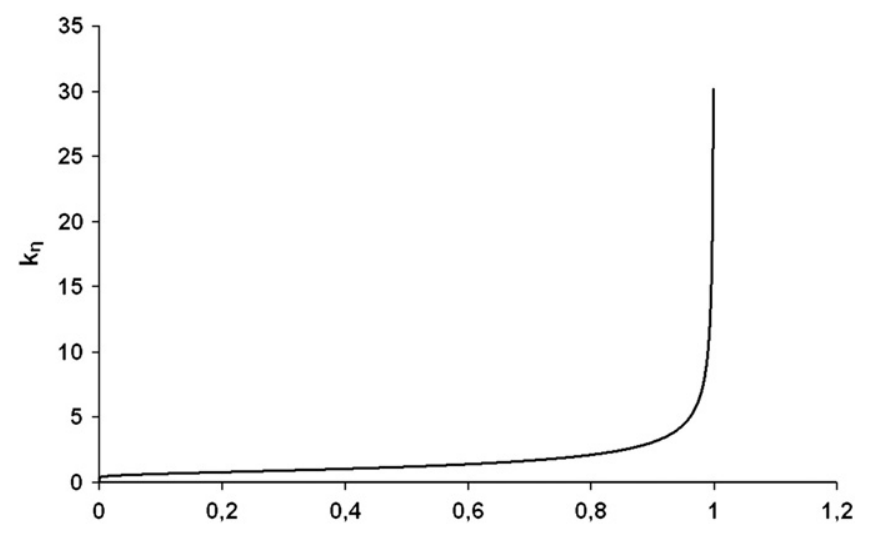

Fig. 2. Plot of the function $k_{\eta}=\sqrt{1 / \log (1 / \eta)}$. 
Table 6

Results of the algorithm for two cases.

\begin{tabular}{|c|c|c|c|c|}
\hline \multirow[b]{2}{*}{ Budget } & \multirow{2}{*}{$\frac{\text { Compactness }}{600 \text { (limited budget) }}$} & \multirow[t]{2}{*}{ Pair effs. } & Compactness & \multirow[t]{2}{*}{ Pair effs. } \\
\hline & & & 6000 & \\
\hline 1 & 12 & 0.863 & 10 & 0.997 \\
\hline 2 & 9 & 0.682 & 18 & 0.999 \\
\hline 3 & 18 & 0.908 & 11 & 0.998 \\
\hline 4 & 10 & 0.766 & 10 & 0.997 \\
\hline 5 & 9 & 0.682 & 17 & 0.9988 \\
\hline 6 & 12 & 0.863 & 12 & 0.9985 \\
\hline 7 & 17 & 0.894 & 12 & 0.9985 \\
\hline 8 & 10 & 0.766 & 9 & 0.996 \\
\hline
\end{tabular}

A similar approach should also hold for the compactness. As a consequence of the increase in the number of spherical and spherical with finger joints in compact mechanisms, another penalty function should be applied to the cost function. The reason for this again lies in the production expenses of advanced kinematic pairs against the primitive ones of the same efficiency. This compactness penalty function is selected as,

$$
k_{L}=\left\{\begin{array}{c}
L \geq 7, \quad k_{L}=|5-L| \\
0 \leq L<7, \quad k_{L}=1+\left(\frac{L}{7}\right)
\end{array}\right.
$$

It should be noted that the compactness penalty function is expressed in two cases, while the rise in the lower bound compactness gives fair penalties, the rise in the higher bound one exacts higher tolls.

There are a few points to mention regarding the selected cost function and expressions immediately following it, given by Eqs. (12-16). The authors here have met a series of problems in this study. Eq. (12) is a simple additive cost function. The softbelly of this expression is that it lacks a sound literature support, since almost no researcher has ever combined entropy with a concept such as complexity. In gathering up Eq. (12), three most important items are added. Drawing a parallel from the field of optimal control, where state and control entities are added through weights, Eq. (12) is formed. Intuitively, the manufacturing cost must rise exponentially as the joint precision goes up. This is the idea behind Eq. (15), which is depicted in Fig. 2. As the joint efficiency approaches 1 , the cost of manufacturing must go to infinity. The weight $k_{\eta}$ is the fruit of a trial and error process for the most representative format in Occam's razor principle. The other is how to represent the complexity of a mechanism. The authors believe that this is the first time that complexity is qualified and quantified, and appears in a cost function in mechanism analysis. Complexity is an on-going discussion in computer science shortly known as the Kolmogorov complexity. But there is no precedence on defining the compactness of a series of links and joints in mechanical engineering. No universal expression unfortunately currently exists.

In the optimization prior to the calculation, the algorithm first asks the amount of budget the user can spend for the manipulator. Later during the calculation process with respect to this budget, the algorithm tries to minimize the cost function by changing the values of compactness and pair efficiencies, provided that the efficiencies do not fall below $\eta=0.4$. Finally by using the results, the user can select the best mechanism for his/her needs either the most compact one or the one with the most efficient pairs. Also note that the user can select an average mechanism between them. In Table 6 the results for two fictitious budget levels are computed for eight runs each.

The resulting $\mathrm{M}=6$ Stewart-Gough platforms are shown in Table 7 with respect to their compactness values. Note that as the mechanism gets compacter, pair efficiencies get larger, and vice versa, when the budget is constant.

\section{Conclusion}

In this study, new classification measures are introduced such as compactness (complexity) and entropy. Their definitions and formulations are elaborated, and a cost function is proposed. Considering compactness and complexity values, examples of robot manipulators that have originated from the same structural groups are created by using exchangeability of kinematic pairs and shown in separate tables. The classification procedure is expanded by using entropy. Genetic algorithms are applied to minimize the proposed cost function for the optimization purposes and different versions of $M=6$ Stewart-Gough platforms are computed.

The mechanism synthesis literature lacks sound work that classifies mechanisms by their increasing complexity and the entropy that waxes as more links and joints are added to the structure. It is believed that this work is unique in its synthesis philosophy. By considering the complexity, entropy and manufacturing costs, the designer would have the upper hand at selecting a prototype to start with. This type of formulation considers the real-life problems, and the authors believe that this would have wide range of applications in the preliminary design stages. If the designer delves into a certain mechanism for the first time, he or she probably would have no idea what the manufacturing costs would be, given the precision and the types of the joints. Starting with a limited budget, a configuration and manufacturing capability, an entropy value might be obtained to weigh a particular configuration against any other. Entropy is the function of efficiency, which may be considered as a rough equivalent of the 
Table 7

Computed $\mathrm{M}=6$ Stewart-Gough platforms.

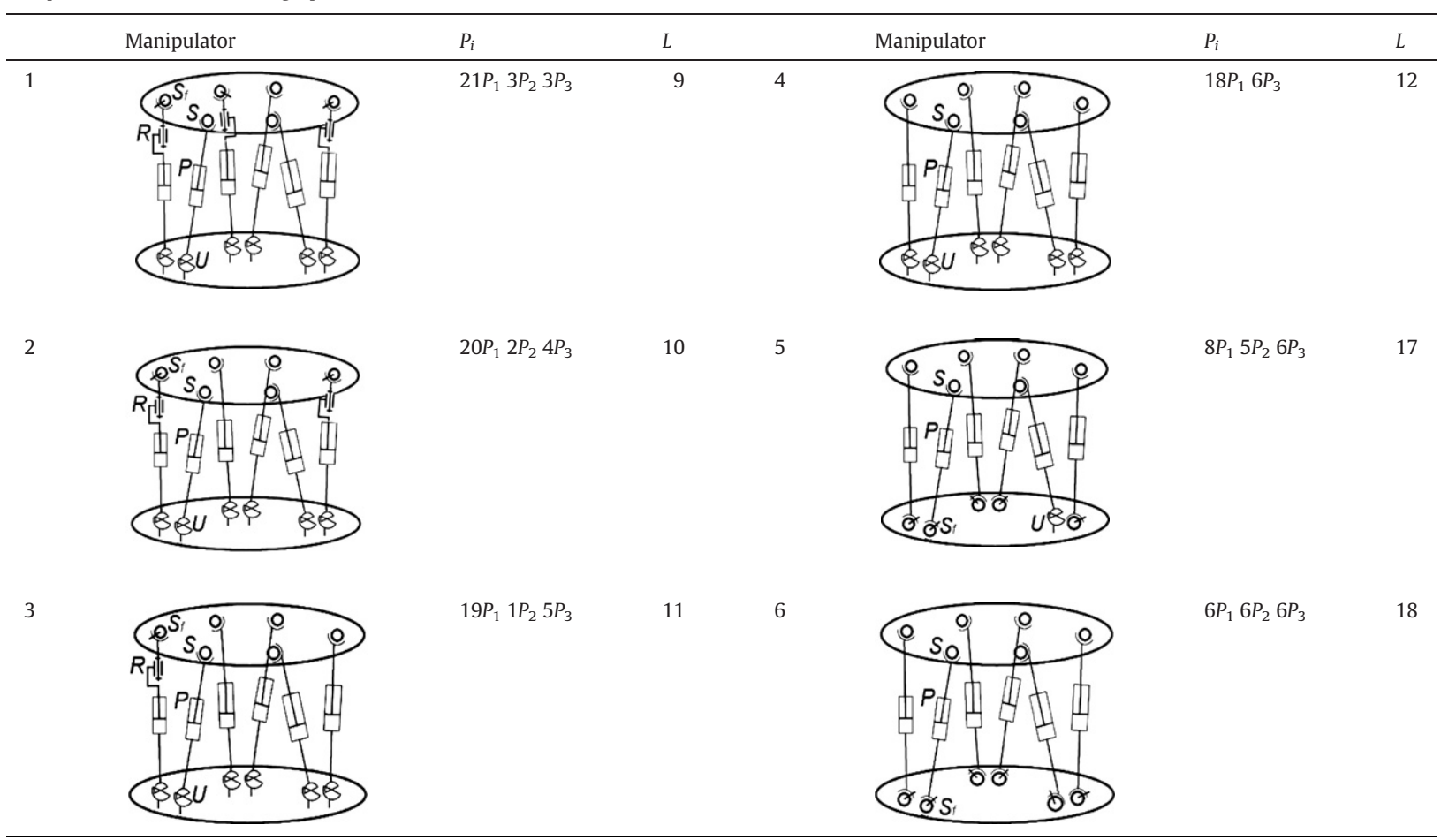

mechanism precision. In this way, entropy is representative of the engineering and resources that are put into it. Another practical use appears at further stages of the design process. Suppose that a certain joint now needs to be slightly more efficient that it was intended. Then the question is what would be the efficiencies of the remaining joints such that the cost, overall entropy and the configuration remain constant.

The authors had experimented on various formats of the complexity-compactness concept, where most have failed in this way or the other. One mode of failure is the inconsistency of a complexity form that does not provide a logical link through different configurations of mechanisms of the same origin. Eqs. (5-6) are in no way complete and all-encompassing, however they are successfully representative of the idea. It is hoped that a later study might focus on a certain structure with individual joints, categorizing joint characteristics in complexity and entropy, setting a bias in the early stages of the optimization toward less questionable ones, which might be considered default configurations.

\section{References}

[1] S. Ozdemir, Measures of uncertainty in power split systems, Mechanism and Machine Theory 42 (2007) 159-167.

[2] A.P. Malushev, Analysis and synthesis of mechanisms from point of view of their structure, Tomsk 91 (1923).

[3] T.S. Mruthyunjaya, Kinematic structure of mechanisms revisited, Mechanism and Machine Theory 38 (2003) $279-320$.

[4] R.I. Alizade, C. Bayram, E. Gezgin, Structural synthesis of Serial Platform Manipulators, Mechanism and Machine Theory (2007).

[5] F. Freudenstein, R.I. Alizade, On the degree of freedom of mechanisms with variable general constraint, IV World IFToMM Congress, England, 1975, pp. 51-56.

[6] R.I. Alizade, On degree of freedom of kinematic chain, Azerbaijan Polytech. Inst., Automation Design of Mechanisms, Manipulators and Robots, Baku, 1988, pp. 3-14.

[7] R.I. Alizade, C. Bayram, Structural synthesis of parallel manipulators, Mechanism and Machine Theory 39 (2004) $857-870$.

[8] T. Balkan, et al., A kinematic structure based classification and compact kinematic equations for six dof industrial robot manipulators, Mechanism and Machine Theory 36 (2001) 817-832.

[9] C.S. Lin, T. Lee, A classification method for input joints of planar five bar mechanisms, Mechanism and Machine Theory 38 (2003) $1307-1322$.

[10] H. Su, C.L. Collins, J.M. McCarthy, Classification of RRSS linkages, Mechanism and Machine Theory 37 (2002) $1413-1433$.

[11] J.J. Cervantes-Sanchez, H.I. Medellin-Castillo, A robust classification scheme for spherical 4R linkages, Mechanism and Machine Theory 37 (2002) $1145-1163$.

[12] S. Ozdemir, J. Schueller, A new hybrid CVT design: CVPSTs, heavy vehicle systems, A series of the International Journal of Vehicle Design 9 (4) (2002) 319-332.

[13] Seungkil Son, Taejung Kim, Sanjay E. Sarma, Alexander Slocum, A hybrid 5-axis CNC milling machine, Precision Engineering 33 (2009) $430-446$.

[14] Kim Hwa Soo, Cho Young Man, Design and modeling of a novel 3-DOF precision micro-stage, Mechatronics 19 (2009) $598-608$.

[15] A. Gonzalez, E. Ottaviano, M. Ceccarelli, On the kinematic functionality of a four-bar based mechanism for guiding wheels in climbing steps and obstacles, Mechanism and Machine Theory 44 (2009) 1507-1523.

[16] Nicolas Binaud, Stéphane Caro, Philippe Wenger, Sensitivity comparison of planar parallel manipulators, Mechanism and Machine Theory 45 (2010) 1477-1490.

[17] T. Munakata, Fundamentals of the New Artificial Intelligence: Beyond Traditional Paradigms, Springer-Verlag, New York, 1998. 